\title{
Generalized Second Law in Cosmology From Causal Boundary Entropy
}

\author{
Ram Brustein \\ Department of Physics, Ben-Gurion University, Beer-Sheva 84105, Israel \\ email: ramyb@bgumail.bgu.ac.il
}

\begin{abstract}
A classical and quantum mechanical generalized second law of thermodynamics in cosmology implies constraints on the effective equation of state of the universe in the form of energy conditions, obeyed by many known cosmological solutions, forbids certain cosmological singularities, and is compatible with entropy bounds. This second law is based on the conjecture that causal boundaries and not only event horizons have geometric entropies proportional to their area. In string cosmology the second law provides new information about non-singular solutions.
\end{abstract}

Typeset using REVTEX 
Cosmological singularities have been investigated, relying on the celebrated singularity theorems of Hawking and Penrose [1], who concluded that if sources in Einstein's equations obey certain energy conditions, cosmological singularities are inevitable. Entropy considerations were brought in only much later, when Bekenstein [2] argued that if the entropy of a visible part of the universe obeys the usual entropy bound from nearly flat space situations [3], certain cosmological singularities are thermodynamically unacceptable. Recently, Veneziano [4] suggested that since a black hole larger than a cosmological horizon cannot form [5], the entropy of the universe is always bounded. This suggestion is related, although not always equivalent, to the application of the holographic principle [6] in cosmology [7] 10].

I propose a concrete classical and quantum mechanical form of a generalized second law (GSL) of thermodynamics in cosmology, valid also in situations far from thermal equilibrium, discuss various entropy sources, such as thermal, geometric and quantum entropy, apply GSL to study cosmological solutions, and show that it is compatible with entropy bounds. GSL allows a more detailed description of how, and if, cosmological singularities are evaded. The proposed GSL is different from GSL for black holes [11], but the idea that in addition to normal entropy other sources of entropy have to be included has some similarities.

That systems with event horizons, such as black holes and a deSitter universe have entropy proportional to the area of their horizon is by now an accepted fact. The proposed GSL is based on the (reasonable) conjecture that causal boundaries and not only event horizons have geometric entropies proportional to their area. However, since the conjecture has not been proved yet, further investigation could reveal that it is incorrect or applies only in special situations. A proof of the conjecture will put our results on a much firmer ground.

The starting point of our classical discussion is the definition of the total entropy of a domain containing more than one cosmological horizon [ []. For a given scale factor $a(t)$, and a Hubble parameter $H(t)=\dot{a} / a$, the number of cosmological horizons within a given comoving volume $V=a(t)^{3}$ is simply the total volume divided by the volume of a single horizon, $n_{H}=a(t)^{3} /|H(t)|^{-3}$ (we will ignore numerical factors of order unity, use units in which $c=1, G_{N}=1 / 16 \pi, \hbar=1$ and discuss only flat, homogeneous, and isotropic 
cosmologies). If the entropy within a given horizon is $S^{H}$, then the total entropy is given by $S=n_{H} S^{H}$. Classical GSL requires that the cosmological evolution, even when far from thermal equilibrium, must obey $d S \geq 0$, in addition to Einstein's equations. In particular,

$$
n_{H} \partial_{t} S^{H}+\partial_{t} n_{H} S^{H} \geq 0
$$

In general, there could be many sources and types of entropy, and the total entropy is the sum of their contributions. If, in some epoch, a single type of entropy makes a dominant contribution to $S^{H}$, for example, of the form $S^{H}=|H|^{\alpha}, \alpha$ being a constant characterizing the type of entropy source, and therefore $S=(a|H|)^{3}|H|^{\alpha}$, eq.(11) becomes an explicit inequality,

$$
3 H+(3+\alpha) \frac{\dot{H}}{H} \geq 0
$$

which can be translated into energy conditions constraining the energy density $\rho$, and the pressure $p$ of (effective) sources. Using the Friedman-Robertson-Walker (FRW) equations,

$$
\begin{aligned}
H^{2} & =\frac{1}{6} \rho \\
\dot{H} & =-\frac{1}{4}(\rho+p) \\
\dot{\rho} & +3 H(\rho+p)=0,
\end{aligned}
$$

and assuming $\alpha>-3$ (which we will see later is a reasonable assumption ) and of course $\rho>0$, we obtain

$$
\begin{array}{lll}
\frac{p}{\rho} \leq \frac{2}{3+\alpha}-1 & \text { for } & H>0 \\
\frac{p}{\rho} \geq \frac{2}{3+\alpha}-1 & \text { for } & H<0 .
\end{array}
$$

Adiabatic evolution occurs when the inequalities in eqs.(田,5) are saturated.

A few remarks about the allowed range of values of $\alpha$ are in order. First, note that the usual adiabatic expansion of a radiation dominated universe with $p / \rho=1 / 3$ corresponds to $\alpha=-3 / 2$. Adiabatic evolution with $p / \rho<-1$, for which the null energy condition is violated would require a source for which $\alpha<-3$. This is problematic since it does not 
allow a flat space limit of vanishing $H$ with finite entropy. The existence of an entropy source with $\alpha$ in the range $\alpha<-2$ does not allow a finite $\partial_{t} S$ in the flat space limit and is therefore suspected of being unphysical. Finally, the equation of state $p=-\rho$ (deSitter inflation), cannot be described as adiabatic evolution for any finite $\alpha$.

Let us discuss in more detail three specific examples. First, as already noted, we have verified that thermal entropy during radiation dominated $(\mathrm{RD})$ evolution can be described without difficulties, as expected. In this case, $\alpha=-\frac{3}{2}$, reproduces the well known adiabatic expansion, but also allows entropy production. The present era of matter domination requires a more complicated description since in this case one source provides the entropy, and another source the energy.

The second case is that of the conjectured geometric entropy $S_{g}$, whose source is the existence of a cosmological horizon [12,13]. The concept of geometric entropy is closely related to the holographic principle, and it has appeared in this connection recently in discussion of cosmological entropy bounds. For a system with a cosmological horizon $S_{g}^{H}$ is given by (ignoring numerical factors of order unity)

$$
S_{g}^{H}=|H|^{-2} G_{N}^{-1}
$$

The equation of state corresponding to adiabatic evolution with dominant $S_{g}$, is obtained by substituting $\alpha=-2$ into eqs.(4.5), leading to $p / \rho=1$ for positive and negative $H$. This equation of state is simply that of a free massless scalar field, also recognized as the two dilaton-driven inflation (DDI) $( \pm)$ vacuum branches of 'pre-big-bang' string cosmology [14] in the Einstein frame. In [4] this was found for the $(+)$ branch in the string frame as an "empirical" observation. In general, for the case of dominant geometric entropy, GSL requires, for positive $H, p \leq \rho$, obtained also by [7] and [8] using a different argument. Note that deSitter inflation (DSI) is definitely allowed. For negative $H$, GSL requires $\rho \leq p$, and therefore forbids, for example, a time reversed history of our universe, or a contracting deSitter universe with a negative constant $H$, unless some additional entropy sources appear.

The third case is that of quantum entropy $S_{q}$, associated with quantum fluctuations. 
This form of entropy was discussed in [15,16]. Specific quantum entropy for a single physical degree of freedom is approximately given by (again, ignoring numerical factors of order unity)

$$
s_{q}=\int d^{3} k \ln n_{k}
$$

where $n_{k} \gg 1$ are occupation numbers of quantum modes [17]. Note that quantum entropy is large for highly excited quantum states, such as the squeezed states obtained by amplification of quantum fluctuations during inflation. Quantum entropy does not seem to be expressible in general as $S_{q}^{H}=|H|^{\alpha}$, since occupation numbers depend on the whole history of the evolution. We will discuss this form of entropy in more detail later, when the quantum version of GSL is proposed.

We would like to show that it is possible to formally define a temperature, and that the definition is compatible with the a generalized form of the first law of thermodynamics. Recall that the first law for a closed system states that $T d S=d E+p d V=(\rho+p) d V+V d \rho$. Let us now consider the case of single entropy source and formally define a temperature $T$, $T^{-1}=\left(\frac{\partial S}{\partial E}\right)_{V}=\frac{\partial s}{\partial \rho}$, since $E=\rho V$ and $S=s V$. Using eqs.(3), and $s=|H|^{\alpha+3}$, we obtain $\frac{\partial s}{\partial \rho}=\frac{\alpha+3}{12}|H|^{\alpha+1}$, and therefore

$$
T=\frac{12}{\alpha+3}|H|^{-\alpha-1}
$$

Note that to ensure positive temperatures $\alpha>-3$, a condition which we have already encountered. Note also that for $\alpha>-1, T$ diverges in the flat space limit, and therefore such a source is suspect of being unphysical, leading to the conclusion that the physical range of $\alpha$ is $-2 \leq \alpha \leq-1$. A compatibility check requires $T^{-1}=\frac{\partial s}{\partial t} / \frac{\partial \rho}{\partial t}$, which indeed yields a result in agreement with (8). Yet another thermodynamic relation $p / T=\left(\frac{\partial S}{\partial V}\right)_{E}$, leads to $p=s T-\rho$ and therefore to $p / \rho=\frac{2}{\alpha+3}-1$ for adiabatic evolution, in complete agreement with eqs.(4, (4). For $\alpha=-2$, eq.(8) implies $T_{g}=|H|$, in agreement with «12 , and for ordinary thermal entropy $\alpha=-3 / 2$ reproduces the known result, $T=|H|^{1 / 2}$.

We turn now to discuss entropy bounds, GSL and cosmological singularities. First, we discuss compatibility of entropy bounds and GSL, and then use GSL to derive a new bound 
relevant to cosmological singularities. Bekenstein [3] suggested that in flat space there is a universal entropy bound on the maximal entropy content in a region containing energy $E$ and of size $L, S<E L$, and then applied this idea to cosmology [2], by choosing the particle horizon $d_{p}=a(t) \int \frac{d t^{\prime}}{a\left(t^{\prime}\right)}$ as $L$. Recently Veneziano [4] argued that since a black hole larger than the horizon cannot form, the largest entropy in a region corresponds to having just one black hole per Hubble volume $H^{-3}$, namely (introducing the Planck mass $M_{p}=G_{N}^{-1 / 2}$ ) that $s \leq M_{p}^{2}|H|$ and

$$
S^{H} \leq M_{p}^{2}|H|^{-2}
$$

This conjecture was further supported in [9]. Perhaps a link between the two distinct entropy bounds can be established by choosing instead of $d_{p}$, the Hubble radius $H^{-1}$ [4,8,9] and since $E_{H}=M_{p}^{2}|H|^{-1}$, the condition $S<E L$ is translated into eq.(9). Note that when applied to non-inflationary cosmology, as done in [2], particle horizon and Hubble radius are about the same and therefore both bounds give similar constraints on $S^{H}$. A consequence of bound (9) is therefore that geometric entropy should always be the dominant source of entropy,

$$
S^{H} \leq S_{g}^{H}
$$

In [9] an example of an expanding and recontracting universe with some matter and a small negative cosmological constant was presented, for which bound (9) seems to be violated. This example involves an epoch in which the causal range is very different from $|H|^{-1}$, and is quite interesting, but its resolution will not affect our conclusions for the cases we are interested in, in which $H^{2}$ is at least as large as $|\dot{H}|$.

Is GSL compatible with entropy bounds? Let us start answering this question by considering a universe undergoing decelerated expansion, that is $H>0, \dot{H}<0$. For entropy sources with $\alpha>-2$, going backwards in time, $H$ is prevented by the entropy bound (10) from becoming too large. This requires that at a certain moment in time $\dot{H}$ has reversed sign, or at least vanished. GSL allows such a transition. Evolving from the past towards the future, and looking at eq.(2) we see that a transition from an epoch of accelerated expansion 
$H>0, \dot{H}>0$, to an epoch of decelerated expansion $H>0, \dot{H}<0$, can occur without violation of GSL. But later we discuss a new bound appearing in this situation when quantum effects are included.

For a contracting universe with $H<0$, and if sources with $\alpha>-2$ exist, the situation is more interesting. Let us check whether in an epoch of accelerated contraction $H<0, \dot{H}<0$, GSL is compatible with entropy bounds. If an epoch of accelerated contraction lasts, it will inevitably run into a future singularity, in conflict with bound (10). This conflict could perhaps have been prevented if at some moment in time the evolution had turned into decelerated contraction with $H<0, \dot{H}>0$. But a brief look at eq.(2) $\dot{H} \leq-\frac{3}{3+\alpha} H^{2}$, shows that decelerated contraction is not allowed by GSL. The conclusion is that for the case of accelerated contraction GSL and the entropy bound are not compatible.

To resolve the conflict between GSL and the entropy bound, we propose adding a missing quantum entropy term $d S_{\text {Quantum }}=-\mu d n_{H}$, where $\mu(a, H, \dot{H}, \ldots)$ is a "chemical potential" motivated by the following heuristic argument. Specific quantum entropy is given by (7), and we consider for the moment one type of quantum fluctuations that preserves its identity throughout the evolution. Changes in $S_{q}$ result from the well known phenomenon of freezing and defreezing of quantum fluctuations. For example, quantum modes whose wavelength is stretched by an accelerated cosmic expansion to the point that it is larger than the horizon, become frozen ("exit the horizon"), and are lost as dynamical modes, and conversely quantum modes whose wavelength shrinks during a period of decelerated expansion ("reenter the horizon"), thaw and become dynamical again. Taking into account this "quantum leakage" of entropy, requires that the first law should be modified as in open systems $T d S=$ $d E+P d V-\mu d N$, as first suggested in [18].

In a universe going through a period of decelerated expansion, containing some quantum fluctuations which have reentered the horizon (e.g., a homogeneous and isotropic background of gravitational waves), physical momenta simply redshift, but since no new modes have reentered, and since occupation numbers do not change by simple redshift, then within 
a fixed comoving volume, entropy does not change. However, if there are some frozen fluctuations outside the horizon "waiting to reenter" then there will be a change in quantum entropy, because the minimal comoving wave number of dynamical modes $k_{m i n}$, will decrease due to the expansion, $k_{\min }(t+\delta t)<k_{\min }(t)$. The resulting change in quantum entropy, for a single physical degree of freedom, is $\Delta s_{q}=\int_{k_{\min }(t+\delta t)}^{k_{\min }(t)} k^{2} d k \ln n_{k}$, and since $k_{\min }(t)=$ $a(t) H(t), \Delta S_{q}=\int_{a(t+\delta t) H(t+\delta t)}^{a(t) H(t)} k^{2} d k \ln n_{k}=-\Delta(a H)^{3} \ln n_{k=a H}$, provided $\ln n_{k}$ is a smooth enough function. Therefore, for $N$ physical degrees of freedom, and since $n_{H}=(a H)^{3}$,

$$
d S_{q}=-\mu N d n_{H}
$$

where parameter $\mu$ is taken to be positive. Obviously, the result depends on the spectrum $n_{k}$, but typical spectra are of the form $n_{k} \sim k^{\beta}$, and therefore we may take as a reasonable approximation $\ln n_{k} \sim$ constant for all $N$ physical degrees of freedom.

We adopt proposal (11) in general,

$$
\begin{aligned}
d S & =d S_{\text {Classical }}+d S_{\text {Quantum }} \\
& =d n_{H} S^{H}+n_{H} d S^{H}-\mu N d n_{H},
\end{aligned}
$$

where $S^{H}$ is the classical entropy within a cosmological horizon. In particular, for the case that $S^{H}$ is dominated by a single source $S^{H}=|H|^{\alpha}$,

$$
\left(3 H+3 \frac{\dot{H}}{H}\right) n_{H}\left(S^{H}-\mu N\right)+\alpha \frac{\dot{H}}{H} n_{H} S^{H} \geq 0 .
$$

Quantum modified GSL (13) allows a transition from accelerated to decelerated contraction. As a check, look at $H<0, \dot{H}=0$, in this case modified GSL requires $3 H\left(S^{H}-\mu N\right) \geq 0$, which, if $\mu N \geq S^{H}$, is allowed. If the dominant form of entropy is indeed geometric entropy, the transition from accelerated to decelerated contraction is allowed already at $|H| \sim M_{p} / \sqrt{N}$. In models where $N$ is a large number, such as grand unified theories and string theory where it is expected to be of the order of 1000, the transition can occur at a scale much below the Planck scale, at which classical general relativity is conventionally expected to adequately describe background evolution. 
If we reconsider the transition from accelerated to decelerated expansion and require that (13) holds, we discover a new bound derived directly from GSL, compatible with, but not relying on, bound (10). Consider the case in which $\dot{H}$ and $H$ are positive, or $H$ positive and $\dot{H}$ negative but $|\dot{H}| \ll H^{2}$, relevant to whether the transition is allowed by GSL. In this case, (13) reduces to $S^{H}-\mu N \geq 0$, that is, GSL puts a lower bound on the classical entropy within the horizon. If geometric entropy is the dominant source of entropy as expected, GSL puts a lower bound on geometric entropy $S_{g}^{H} \geq \mu N$, which yields an upper bound on $H$,

$$
H \leq \frac{M_{p}}{\sqrt{N}}
$$

The scale that appeared previously in the resolution of the conflict between entropy bounds and GSL for a contracting universe has reappeared in (14), and remarkably, (14) is the same bound obtained in [2] using different arguments. Bound (14) forbids a large class of singular homogeneous, isotropic, spatially flat cosmologies by bounding their curvature.

An interesting study case is 'pre-big-bang' string cosmology [14]. In this scenario the evolution of the universe starts from a state of very small curvature and string coupling, undergoes a phase of dilaton-driven inflation (DDI) which joins smoothly standard radiation dominated (RD) cosmology, thus giving rise to a singularity free inflationary cosmology. The graceful exit transition from DDI to RD, has been studied intensely [19], with the following scenario emerging, first classical corrections limit the curvature by trapping the universe in an algebraic fixed point [20] a linear dilaton deSitter solution, and then quantum corrections limit the string coupling, and end the transition [21,22]. Modified GSL supports this exit scenario, clarifies the conditions for the existence of the algebraic fixed point, determines new energy conditions, and constrains sources required to complete a graceful exit transition.

We present here the case of dominant $S_{g}$. A candidate geometric entropy is given by the analog of eq.(6) 四 by substituting $M_{p}^{2}=e^{-\phi} M_{S}, M_{S}$ being the (constant) string mass and $\phi$ is the dilaton, $S^{H}=e^{-\phi} H^{-2}$. The expression for $n_{H}$ is unchanged. Condition (2) now reads $3 H+\frac{\dot{H}}{H}-\dot{\phi} \geq 0$. Using $\dot{\bar{\phi}}=\dot{\phi}-3 H$, we obtain $\frac{\dot{H}}{H}-\dot{\bar{\phi}} \geq 0$, leading, by using one of string cosmology equations of motion $\bar{\sigma}-2 \dot{H}+2 H \dot{\bar{\phi}}=0$ [23], for $H>0$, which is the 
natural choice for pre-big-bang phase, to the energy condition $\bar{\sigma} \geq 0$ [24]. An immediate consequence is that if $\dot{H}$ vanishes, then $\dot{\bar{\phi}}<0$, so an algebraic fixed point 20] necessarily

has to occur for $\dot{\bar{\phi}}<0$. The same conclusion was reached in [- [], and previously in [20,21]. Further investigation is required clarify the correct comparison to the analysis in ordinary FRW cosmology.

\section{ACKNOWLEDGMENTS}

Work supported in part by the Israel Science Foundation. It is a pleasure to thank R. Madden and G. Veneziano for useful discussions and helpful suggestions and comments, S. Foffa and R. Sturani for discussions about string cosmology, D. Eichler and J. Donoghue for discussions, and J. Bekenstein, R. Easther, N. Kaloper and A. Linde for comments on the manuscript. 


\section{REFERENCES}

[1] S. Hawking and R. Penrose, Proc. Roy. Soc. Lond. A314, 529 (1970).

[2] J. Bekenstein, Int. J. Theor. Phys. 28, 967 (1989).

[3] J. Bekenstein, Phys. Rev. D23 , 287 (1981); Phys. Rev. D49, 1912 (1994).

[4] G. Veneziano, Phys. Lett. B454, 22 (1999).

[5] B. J. Carr and S. W. Hawking, MNRAS 168, 399 (1974).

[6] G. 't Hooft, gr-qc/9310026; L. Susskind, J. Math. Phys. 36 (1995) 6377.

[7] W. Fischler and L. Susskind, hep-th/9806039.

[8] R. Easther and D. Lowe, Phys. Rev. Lett. 82, 4967 (1999).

[9] N. Kaloper and A. Linde, Phys. Rev. D60, 103509 (1999).

[10] D. Bak and S. Rey, Class. Quant. Grav. 17, L1 (2000) and hep-th/9902173; A. Biswas, J. Maharana and R. Pradhan, Phys. Lett. B462, 243 (1999); R. Dawid, Phys. Lett. B451, 19 (1999); S. Rama and T. Sarkar, Phys. Lett. B450 , 55 (1999).

[11] J. Bekenstein, Phys. Rev. D7, 2333 (1973); Phys. Rev. D9, 3292 (1974).

[12] G. Gibbons and S. Hawking, Phys. Rev. D15, 2738 (1977).

[13] M. Srednicki, Phys. Rev. Lett. 71, 666 (1993).

[14] G. Veneziano, Phys. Lett. B265, 287 (1991); M. Gasperini and G. Veneziano, Astropart. Phys. 1, 317 (1993).

[15] R. Brandenberger, V. Mukhanov and T. Prokopec, Phys. Rev. Lett. 69, 3606 (1992); Phys. Rev. D48, 2443 (1993).

[16] M. Gasperini and M. Giovannini, Phys. Lett. B301, 334 (1993); Class. Quant. Grav. 10, L133 (1993). 
[17] See [15 for modification of (7) for the case $n_{k} \sim 1$.

[18] I. Prigogine et al., Gen. Rel. and Grav. 21, 767 (1989).

[19] R. Brustein and G. Veneziano, Phys. Lett. B329, 429 (1994); N. Kaloper, R. Madden and K.A. Olive, Nucl. Phys. B452, 677 (1995).

[20] M. Gasperini, M. Maggiore and G. Veneziano, Nucl. Phys. B494, 315 (1997).

[21] R. Brustein and R. Madden, Phys. Lett. B410, 110 (1997); Phys. Rev. D57, 712 (1998).

[22] A. Buonanno, et al., JHEP 9801, 004 (1998).

[23] R. Brustein and R. Madden, JHEP 9907, 006 (1999).

[24] First found by R. Madden. 